\title{
Peculiarities of the magnetocaloric effect in FeRh-based alloys in the vicinity of the first order magnetic phase transition
}

\author{
Radel Gimaev ${ }^{1,2,}{ }^{*}$, Vladimir Zverev ${ }^{3}$, Yury Spichkin ${ }^{1,2}$, Alexander Tishin ${ }^{1,2,3}$ and Takafumi Miyanaga ${ }^{4}$ \\ ${ }^{1}$ Advanced Magnetic Technologies and Consulting LLC, 142191 Troitsk, Moscow, Russia \\ ${ }^{2}$ National Research Centre "Kurchatov Institute", 123182, Moscow, Russia \\ ${ }^{3}$ Faculty of Physics, M.V. Lomonosov Moscow State University, 119991 Moscow, Russia \\ ${ }^{4}$ Department of Mathematics and Physics, Hirosaki University, Hirosaki, Aomori, 036-8561, Japan
}

\begin{abstract}
Medical applications of magnetocaloric effect (MCE) require possibility for precision shift of a temperature of the magnetic phase transition at the same MCE value and minimize irreversibility. Thus, detail dynamic MCE investigation of such alloys with non-toxic biocompatible dopants need to be done. In present work, the giant magnetocaloric effect, which is observed in the whole family of Fe-Rh alloys, has been investigated in Pd-doped samples in slowly cycled magnetic fields of up to $1.8 \mathrm{~T}$ in magnitude for a range of temperatures, $250 \mathrm{~K}<\mathrm{T}<350 \mathrm{~K}$. The shift of the ferromagnetic/antiferromagnetic transition temperature down towards room temperature and the decrease in the MCE have been observed in these alloys in comparison with a quasi-equiatomic FeRh alloy. The measurements have also shown an asymmetric behaviour of the first order magnetic phase transition with respect to whether the transition is traversed by heating from lower temperatures or cooling from above. These peculiarities have been explained in the framework of the ab-initio density functional theory-based disordered local moment theory of the MCE. The results have been compared with the those for the non-doped FeRh alloy. Thus features of the first order magnetic phase transition that these alloys have in common have been revealed which enable some predictions to be made appropriate for practical applications.
\end{abstract}

\section{Introduction}

Conventional nearly equiatomic stoichiometric Fe$\mathrm{Rh}$ alloys exhibit a giant magnetocaloric effect (MCE) but have some limitations for widespread use in magnetic refrigeration technology. Among these are the sharp ferromagnetic/antiferromagnetic transition, the relatively high transition temperature and, of course, the high cost of $\mathrm{Rh}$ as a constituent of the working body of a refrigerator [1-4]. The obvious idea is to dope the pure alloy having CsCl-type structure with some elements so that the crucial parameters are adjusted to more suitable values. First attempts in making indirect $\Delta S$ measurements during the $1^{\text {st }}$ order magnetic phase transition in Fe-Rh alloys doped with $\mathrm{Pd}$, Pt and Ir have been performed in $[5,6]$. The authors notice the large contribution of the magnetic entropy change $(\sim 10 \mathrm{~J} / \mathrm{kg}$ $\mathrm{K})$ to the full $\Delta S$ change $(\sim 14 \mathrm{~J} / \mathrm{kg} \mathrm{K})$ during the transition at magnetic field up to $1.2 \mathrm{~T}$.

Doping Fe-Rh alloys with $\mathrm{Pd}, \mathrm{Cu}, \mathrm{Co}$, Ni etc. leads to the shift of the phase transition towards low temperatures [7-11] which is important for medical purposes for example. The idea of this work is to move phase transition temperature around of human body temperature region at saving MCE value. Increase of Pd content is the reason of the lattice parameter increase [11]. For practical applications the shift of the MCE maximum during magnetization and demagnetization processes should be considered [12].

The importance of doping is to significantly increase the Refrigerant capacity (RC) value $[10,13]$. Adding some $3 \mathrm{~d}$ or $4 \mathrm{~d}$ transition metals changes the chemical order in the alloy drastically which leads to a significant broadening of the phase transition transformation range. Although doping decreases the MCE value, the peak broadens which leads to an overall increase of RC.

Nowadays there are just a few promising materials which undergo a $1^{\text {st }}$ order antiferromagnetic to ferromagnetic (AFM-FM) transition and only FeRh has the transition occurring close to room temperature [14]. The idea to use this material in magnetic refrigerators was first proposed by [15]. Later on discussion about the creation of a magnetic refrigerator which employs the $1^{\text {st }}$ order AFM-FM transition were published [16,17].

The high cost of Rh, however, drives research into other areas for application of this material where the cost problem is not so crucial. A good example is medicine where polycrystalline materials might be suitable. Additionally, Fe-Rh alloys and $\mathrm{Pd}$ are non-toxic biocompatible materials.

\footnotetext{
Corresponding author: gimaev@amtc.org
} 


\section{Materials and methods}

The $\mathrm{Fe}_{49.7} \mathrm{Rh}_{47.4} \mathrm{Pd}_{2.9}$ and $\mathrm{Fe}_{48.9} \mathrm{Rh}_{46.8} \mathrm{Pd}_{4.9}$ alloys were prepared using plasma arc melting in $\mathrm{Ar}$ atmosphere (pressure was $0.09 \mathrm{MPa}$ ). Samples were annealed in vacuum at $1273 \mathrm{~K}$ during $48 \mathrm{~h}$ and were quenched by cooling into water.

Direct magnetocaloric effect (MCE) measurements were performed using the MagEq MMS 801 (AMT\&C LLC) system which has been described in [18-20]. Direct measurements were conducted in the same way as reported ones previously [20].

The theoretical description of MCE properties of $\mathrm{Fe}_{49,7} \mathrm{Rh}_{47,4} \mathrm{Pd}_{2.9}$ and $\mathrm{Fe}_{48,9} \mathrm{Rh}_{46,8} \mathrm{Pd}_{4.9}$ alloys was made using the ab-initio disordered local moment theory $[21,22]$.

In an ideal stoichiometrically ordered $\mathrm{Fe}-\mathrm{Rh}$ alloy with bec structure, each iron atom is surrounded by 8 rhodium atoms as nearest neighbors and 6 iron atoms as second-order neighbors. Interactions between $\mathrm{Fe}-\mathrm{Fe}$ atoms in the bcc structure of $\mathrm{Fe}-\mathrm{Rh}$ alloys favor the onset of AFM ordering, while the FM ordering is caused by interaction with the nearest neighbors, i.e. Rh atoms. The ideal order in the bcc structure of Fe-Rh-based alloys is not achieved in real alloys. The phase analysis of the $\mathrm{Fe}_{50} \mathrm{Rh}_{50}$ alloy shows $[20,22]$ that during the annealing and subsequent cooling $1-2 \%$ of rhodium atoms are replaced by iron atoms and vice versa, which can not be eliminated by subsequent heat treatment. Thus, an insignificant disordering is introduced into the system: some of $\mathrm{Rh}$ atoms are replaced by $\mathrm{Fe}$ atoms and the nearest neighbors of these Fe atoms are Fe atoms. Such regions in which the nearest neighbors are two Fe atoms have a strong FM interaction, which is manifested in the bcc structure of iron. Thus, the appearance of a small number of nearest Fe-Fe neighbors (1-2\%) leads to a strong effect on the AFM-FM magnetic transition.

Theoretical consideration of substitution defects is based on the model described in detail in [22]. Within the framework of this model, two sublattices of $\mathrm{Fe}$ and $\mathrm{Rh}$ atoms are considered in the crystalline bcc lattice of $\mathrm{Fe}-\mathrm{Rh}$ alloys with close to equiatomic compositions. The first sublattice (sublattice A) has local locations for (a) Fe atoms, but Rh atoms occupy a small percentage (x) of these locations (real substitution occurs in real alloys) [22]. The second sublattice (sublattice B) is a sublattice of the arrangement of (b) Rh atoms, in which a small percentage (y) of $\mathrm{Fe}$ atoms also lies. When considering the equiatomic $\mathrm{Fe}-\mathrm{Rh}$ alloy in the model of two sublattices (in the sublattice A, the main atom is Fe with the presence of a small number of $x$ Rh atoms, in the sublattice $\mathrm{B}$ the main atom is $\mathrm{Rh}$ with the presence of a small number of $\mathrm{y}$ atoms $\mathrm{Fe}$ ), the $\mathrm{Fe}-\mathrm{Rh}$ alloy can be written in the form $\mathrm{Fe}_{100-\mathrm{x}} \mathrm{Rh}_{\mathrm{x}} \mathrm{Rh}_{100-\mathrm{y}} \mathrm{Fe}_{\mathrm{y}}$. In this notation, the concentration of Fe atoms in the alloy can be written down as $c=\frac{100-x+y}{2}$, and long order parameter as $=\frac{100-y}{100}$.

For the composition of the Fe-Rh alloy, with certain values of $\mathrm{x}$ and $\mathrm{y}$, the free energy of the alloy can be written as [22]:

$$
\begin{gathered}
F(c, y, H, T)=U\left(c, y, m_{f}, m_{a}\right)-T\left(S_{\text {маг }}\left(m_{f}, m_{a}, T\right)+\right. \\
\left.S_{\text {реш }}(T)\right)-H m_{f}
\end{gathered}
$$

where $U$ is the internal energy, $S_{m a g}$ is the magnetic part of the entropy, $S_{\text {latt }}$ is the entropy of the vibrations of the crystal lattice, $\mathrm{H}$ is the magnetic field, $\mathrm{m}_{\mathrm{f}}$ is the PM ordering parameter (proportional to the magnetization), $\mathrm{m}_{\mathrm{a}}$ is the AFM ordering parameter. The parameters $\mathrm{m}_{\mathrm{a}}$ and $m_{f}$ give the degree of FM and AFM ordering in the alloy, so for the pure AFM ordering the parameters $\mathrm{m}_{\mathrm{a}}=$ $1, \mathrm{~m}_{\mathrm{f}}=0$, for pure $\mathrm{FM}$ ordering $\mathrm{m}_{\mathrm{a}}=0, \mathrm{~m}_{\mathrm{f}}=1$. In the high-temperature paramagnetic region, both parameters $\mathrm{m}_{\mathrm{a}}$ and $\mathrm{m}_{\mathrm{f}}$ are equal to zero.

\section{Results and discussion}

The magnetocaloric properties of Pd-doped Fe-Rh alloys $\mathrm{Fe}_{49,7} \mathrm{Rh}_{47,4} \mathrm{Pd}_{2.9}$, and $\mathrm{Fe}_{48,9} \mathrm{Rh}_{46,8} \mathrm{Pd}_{4.9}$ are studied. It is known that adding Pd leads to a decrease of the FM/AFM transition temperature bringing it closer to room temperature $[5,6,9,11,13,23,24]$. Thus, the study of such materials is promising from the practical point of view as it allows materials to be prepared which have desirable transition temperature values. In addition, doping the alloys with Pd does not lead to any significant MCE decrease. To ascertain whether the irreversibility effect is also present in these alloys we prepared two samples with different amounts of Pd. As with the Fe-Rh sample we measured the MCE on both heating and cooling through the phase transition. It should be noted, that $\Delta \mathrm{T}(\mathrm{H})$ measurements were performed at magnetic field change rates of $1 \mathrm{~T} / \mathrm{s}-5 \mathrm{~T} / \mathrm{s}$ but since they were practically identical here we present the ones obtained at $1 \mathrm{~T} / \mathrm{s}$.

The suppression of the transition temperature, $T_{t r}$, can be understood from the DLM-DFT (disordered local moments density functional theory) based model. As described in [22] an increase of the number of Fe-Fe nearest neighbors in Fe-Rh sharply depresses $T_{t r}$. This happens if $\mathrm{Fe}-\mathrm{Rh}$ is made marginally iron-rich and/or if the $\mathrm{CsCl}$ chemical order is incomplete, i.e. a small number of positions on the $\mathrm{Rh}$ sub-lattice in the $\mathrm{CsCl}$ crystal structure are occupied by Fe atoms. Our DFT calculations reported in [20] based on model presented in $[21,22]$ showed that addition of $\mathrm{Pd}$ enhances this process. They indicate that $\mathrm{Pd}$ atoms are liable to substitute for $\mathrm{Fe}$ and $\mathrm{Rh}$ atoms with roughly equal tendency. The net outcome is that Pd-doped Fe-Rh alloys have an increased likelihood of some $\mathrm{Fe}$ atoms residing on the ' $\mathrm{Rh}$ ' sub-lattice as a consequence of the added $\mathrm{Pd}$. This is consistent with the reported decrease in $T_{t r}$. Incidentally calculations for Pt-doped Fe- Rh alloys showed the reverse - Pt atoms preferentially displaced $\mathrm{Rh}$ atoms and reduced the tendency of the iron atoms to migrate to the 'Rh' sub-lattice. $T_{t r}$ in this case would expected to increase with Pt-doping [13].

Fig. 1 shows the $\Delta T$ temperature dependences for (a) $\mathrm{Fe}_{49,7} \mathrm{Rh}_{47,4} \mathrm{Pd}_{2.9}$ and (b) $\mathrm{Fe}_{48,9} \mathrm{Rh}_{46,8} \mathrm{Pd}_{4.9}$ (magnetic field 
changed up to $1.8 \mathrm{~T}$ ). The shift of the AFM-FM transition towards lower temperatures than that of $\mathrm{Fe}_{50.4} \mathrm{Rh}_{49.6}[20,25]$ was $25 \mathrm{~K}$ and $30 \mathrm{~K}$ for $\mathrm{Fe}_{49,7} \mathrm{Rh}_{47,4} \mathrm{Pd}_{2.9}$ and $\mathrm{Fe}_{48,9} \mathrm{Rh}_{46,8} \mathrm{Pd}_{4.9}$, respectively. In the present work we obtained transition temperatures $298 \mathrm{~K}$ and $293 \mathrm{~K}$ for $\mathrm{Fe}_{49,7} \mathrm{Rh}_{47,4} \mathrm{Pd}_{2,9}$ and $\mathrm{Fe}_{48,3} \mathrm{Rh}_{46,8} \mathrm{Pd}_{4,9}$, respectively, which were very close to room temperature values required in magnetic refrigeration technology. The shift of the transition temperature was in good agreement with previous results $[5,6,9,11,13,23,24]$. The transition in the Pd-doped samples spread over a wider range in comparison with $\mathrm{Fe}_{50.4} \mathrm{Rh}_{49.6}$ [20,25]: full width at half maximum (FWHM) $10 \mathrm{~K}$ for $\mathrm{Fe}_{50,4} \mathrm{Rh}_{49,6}$ and 12.4 $\mathrm{K}$ for $\mathrm{Fe}_{49.7} \mathrm{Rh}_{47.4} \mathrm{Pd}_{2.9}$ and $\mathrm{Fe}_{48.9} \mathrm{Rh}_{46.8} \mathrm{Pd}_{4.9}$ (Fig. 2). There was a decrease of the MCE values which was approximately $-6 \mathrm{~K}$ at the maximum value. The same peculiarities were observed during the FM-AFM transition on cooling as with $\mathrm{Fe}_{50.4} \mathrm{Rh}_{49.6}[20,25]$ but with smaller MCE values distributed with a narrower peak in comparison with the results for the AFM-FM transition.

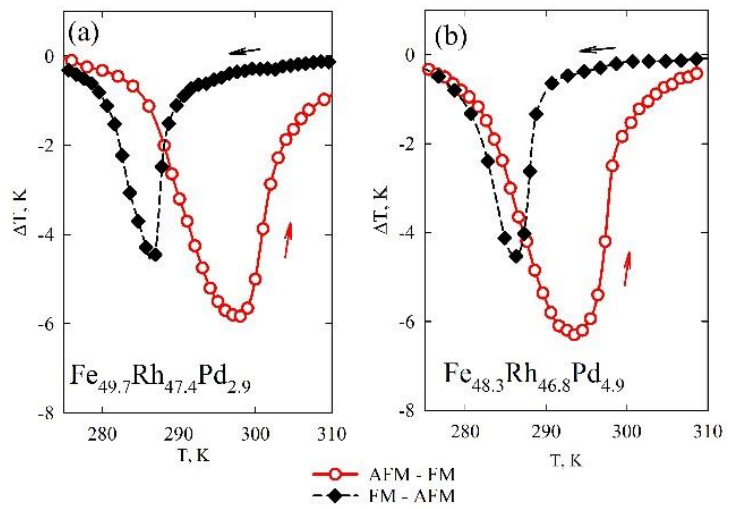

Fig. 1. $\Delta T(T)$ dependences for the bulk Pd-doped FeRh alloy samples $\mathrm{Fe}_{49,7} \mathrm{Rh}_{47,4} \mathrm{Pd}_{2.9} \quad$ (a) and $\mathrm{Fe}_{48,9} \mathrm{Rh}_{46,8} \mathrm{Pd}_{4.9}$ (b) on heating (open red circles) and cooling (filled black diamonds). Magnetic field changed up to $1.8 \mathrm{~T}$. The arrows show the direction of the temperature change.

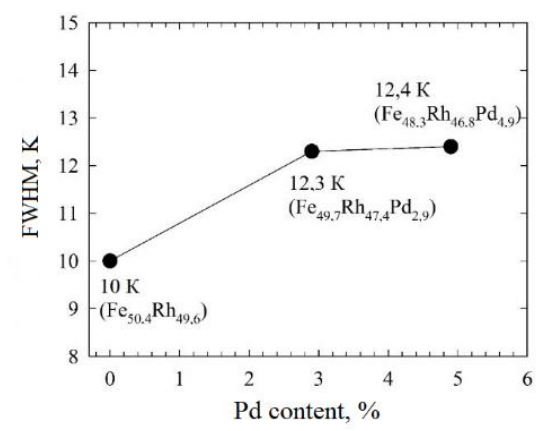

Fig. 2. FWHM for different Pd content in FeRh alloy.

The variation of $\Delta T$ as the field was cycled in $\mathrm{Fe}_{49,7} \mathrm{Rh}_{47,4} \mathrm{Pd}_{2.9}$ and $\mathrm{Fe}_{48,9} \mathrm{Rh}_{46,8} \mathrm{Pd}_{4.9}$ displayed similar behavior to that of the FeRh alloy [20,25]. The results of such dynamic $\Delta T$ measurements for one full cycle of the magnetic field in the range $-1.8 \mathrm{~T}-+1.8 \mathrm{~T}$ for the alloy $\mathrm{Fe}_{48,9} \mathrm{Rh}_{46,8} \mathrm{Pd}_{4.9}$ are presented in Fig. 3. We have obtained $\Delta T(\mathrm{H})$ curves for 40 different temperatures in the range $270 \mathrm{~K}-310 \mathrm{~K}$. Here we present three of them: where MCE reached its maximum (Fig. 3 (b) and (e)), and at temperatures with dominating AFM (Fig. 3 (a) and (d)) and FM phases, respectively (Fig. 3 (c) and (f)). The analogous $\Delta T(\mathrm{H})$ curves for $\mathrm{Fe}_{49,7} \mathrm{Rh}_{47,4} \mathrm{Pd}_{2.9}$ showed the same features and thus are not shown.

$$
\mathrm{Fe}_{48.3} \mathrm{Rh}_{46.8} \mathrm{Pd}_{4.9} \text {, heating from AFM }
$$
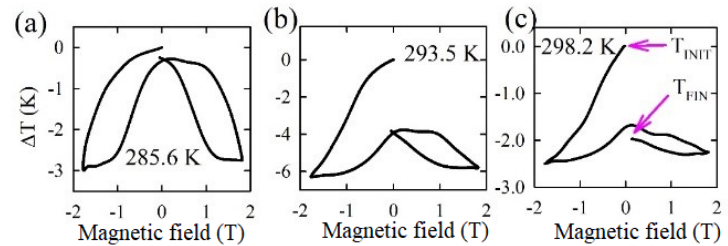

$\mathrm{Fe}_{48.3} \mathrm{Rh}_{46.8} \mathrm{Pd}_{4.9}$, cooling from FM (d)

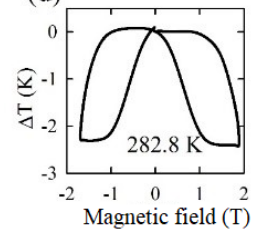

(e)

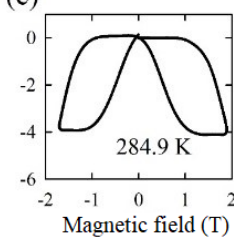

(f)

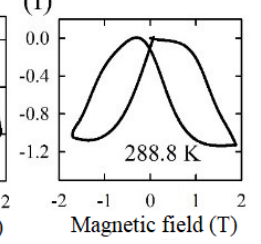

Fig. 3. $\Delta \mathrm{T}(\mathrm{H})$ dependences for $\mathrm{Fe}_{48,9} \mathrm{Rh}_{46,8} \mathrm{Pd}_{4.9}$ obtained at three different temperature values in the vicinity of the phase transition on heating from AFM phase (a)-(c) and cooling from FM one (d)-(f). The arrows in (c) show the initial $\mathrm{T}_{\text {INIT }}$ and final $\mathrm{T}_{\mathrm{FIN}}$ temperatures of the sample during the full magnetic field cycle.

The analysis showed that the observed features in the experimental dependences $\Delta \mathrm{T}(\mathrm{H})$ and $\Delta \mathrm{T}(\mathrm{T})$ can be due to the presence of minor crystal replacement defects in which up to $2 \%$ of $\mathrm{Fe}$ atoms are replaced by $\mathrm{Rh}$ atoms and vice versa.

It is important to note that the change in chemical composition and addition of $\mathrm{Pd}$ did not alter the 'irreversibility' features observed in the FeRh sample. The difference between initial and final temperatures after one full cycle of magnetic field $\left(\mathrm{T}_{\mathrm{FIN}}-\mathrm{T}_{\mathrm{INIT}}\right)$ was observed when heating through the AFM-FM transition (Fig. 3 (a)-(c)) but was not present when cooling through the FM-AFM transition (Fig. 3 (d)-(f)). The value of $\left(\mathrm{T}_{\mathrm{FIN}}-\mathrm{T}_{\mathrm{INIT}}\right)$ depended on temperature and this dependence was similar to the one presented in Fig. 4 in ref. [20].

\section{Conclusion}

Direct MCE temperature and field measurements in FeRh-based alloys $\mathrm{Fe}_{49,7} \mathrm{Rh}_{47,4} \mathrm{Pd}_{2.9}$ and $\mathrm{Fe}_{48,9} \mathrm{Rh}_{46,8} \mathrm{Pd}_{4.9}$ showed common features of their MCE in the vicinity of the $1^{\text {st }}$ order magnetic phase transition which each alloy possesses. Each material showed a non-symmetric character of its magnetocaloric properties on heating and cooling which was demonstrated by the presence/absence of the 'irreversibility' of the sample's temperature to its initial value during a full cycle of the magnetic field on heating/cooling. Doping samples with $\mathrm{Pd}$ did not lead to the disappearance of this effect. Increasing the amount of Pd decreased the MCE value and shifted the phase transition temperature down 
towards room temperature (down to $293 \mathrm{~K}$ in $\mathrm{Fe}_{48,9} \mathrm{Rh}_{46,8} \mathrm{Pd}_{4.9}$ for the AFM-FM transition on heating).

At the same time it was shown that doping samples with Pd leads to increasing of FWHM of $\Delta T(T)$ dependence (from $10 \mathrm{~K}$ for FeRh alloy up to $12.4 \mathrm{~K}$ for Pd doped samples) and the relative cooling power ( $\mathrm{RCP})$ value is not changes. Thus, the Pd doping allows tuning of phase transition temperature without decreasing of RCP. This finding enables the appropriate positioning of the maximum MCE value for applications. Unlike ferromagnetic materials such as Gd, the MCE completely disappears on both sides of the phase transition point for temperatures more than 15-20 K away from the transition temperature. Consequently, in addition to their cost limitations these materials cannot be considered for conventional magnetic refrigeration technology over a wide enough range of temperatures. They are, however, promising choices for use in medical applications [26].

The authors thankful to Prof. Julie B. Staunton and Prof. Alexander Shtil for useful discussions. The authors thank the Ministry of Science and Education of Russia for financial support of the work (Federal target program "Research and development in priority areas of development of Russia's scientific and technological complex for 2014-2020", grant identification RFMEFI60417X0197). Work in Advanced Magnetic Technologies and Consulting, LLC is supported by Skolkovo Foundation, Russia.

\section{References}

1. S. A. Nikitin, G. Myalikgulyev, A. M. Tishin, M. P. Annaorazov, K. A. Asatryan, and A. L. Tyurin, Phys. Lett. A 148, 363 (1990).

2. M. P. Annaorazov, K. A. Asatryan, S. A. Nikitin, A. M. Tishin, and A. L. Tyurin, Pisma Zh. Tekh. Phys 12, (1991).

3. M. P. Annaorazov, K. A. Asatryan, G. Myalikgulyev, S. A. Nikitin, A. M. Tishin, and A. L. Tyurin, Cryogenics 32, 867 (1992).

4. M. P. Annaorazov, H. M. Güven, and K. Bärner, J. Alloys Compd. 397, 26 (2005).

5. J. S. Kouvel and C. C. Hartelius, J. Appl. Phys. 33, 1343 (1962).

6. J. S. Kouvel, J. Appl. Phys. 37, 1257 (1966).

7. N. V. Baranov, S. V. Zemlyanski, and K. Kamenev, in Itinerant Electron Magn. Fluct. Eff., edited by D. Wagner, W. Brauneck, and A. Solontsov (Springer Netherlands, 1998), pp. 345-351.

8. N. V. Baranov and E. A. Barabanova, J. Alloys Compd. 219, 139 (1995).

9. A. M. Chirkova, A. S. Volegov, D. S. Neznakhin, E. A. Stepanova, and N. V. Baranov, Solid State Phenom. 190, 299 (2012).
10. M. Manekar and S. B. Roy, J. Phys. Appl. Phys. 44, 242001 (2011).

11. K. Nishimura, Y. Nakazawa, L. Li, and K. Mori, Mater. Trans. 49, 1753 (2008).

12. Y. S. Koshkid'ko, J. Ćwik, T. I. Ivanova, S. A. Nikitin, M. Miller, and K. Rogacki, J. Magn. Magn. Mater. Complete, 234 (2017).

13. R. Barua, F. Jiménez-Villacorta, and L. H. Lewis, J. Appl. Phys. 115, 17A903 (2014).

14. J.-U. Thiele, T. Hauet, and O. Hellwig, Appl. Phys. Lett. 92, 242502 (2008).

15. S. A. Nikitin, G. Myalikgulyev, A. M. Tishin, M. P. Annaorazov, K. A. Asatryan, and A. L. Tyurin, Avtorskoe svidetel’stvo-№ SU 1746162 A1 (in Russia) (7 July 1992).

16. M. P. Annaorazov, M. Ünal, S. A. Nikitin, A. L. Tyurin, and K. A. Asatryan, J. Magn. Magn. Mater. 251, 61 (2002).

17. M. P. Annaorazov, S. A. Nikitin, A. L. Tyurin, S. A. Akopyan, and R. W. Myndyev, Int. J. Refrig. 25, 1034 (2002).

18. Y. I. Spichkin, A. M. Tishin, D. B. Kopeliovich, and A. Y. Malyshev, in (Third IIF-IIR International Conference on Magnetic Refrigeration at Room Temperature (Thermag III), Des Moines, Iowa, USA, 2009), pp. 175-180.

19. Y. I. Spichkin and R. R. Gimaev, Int. J. Refrig. 37, 230 (2014).

20. V. I. Zverev, A. M. Saletsky, R. R. Gimaev, A. M. Tishin, T. Miyanaga, and J. B. Staunton, Appl. Phys. Lett. 108, 192405 (2016).

21. B. L. Gyorffy, A. J. Pindor, J. Staunton, G. M. Stocks, and H. Winter, J. Phys. F Met. Phys. 15, 1337 (1985).

22. J. B. Staunton, R. Banerjee, M. dos S. Dias, A. Deak, and L. Szunyogh, Phys. Rev. B 89, 054427 (2014).

23. S. Yuasa, Y. Otani, H. Miyajima, and A. Sakuma, IEEE Transl. J. Magn. Jpn. 9, 202 (1994).

24. T. Zhou, M. K. Cher, L. Shen, J. F. Hu, and Z. M. Yuan, Phys. Lett. A 377, 3052 (2013).

25. R. R. Gimaev, V. I. Zverev, A. M. Saletsky, A. M. Tishin, T. Miyanaga, and J. B. Staunton, in Proc 7th Int C Magn Refrig Room Temp IIFIIR (International Institute of Refrigeration (IIR), 2016), pp. 279-286.

26. A. M. Tishin, Y. I. Spichkin, V. I. Zverev, and P. W. Egolf, Int. J. Refrig. 68, 177 (2016). 